\title{
Correction to: Breast cancer, diabetes mellitus and glucagon-like peptide-1 receptor toward exploring their possible associations
}

\author{
Naoko Hashimoto Takigami ${ }^{1,2}$. Shimpei Kuniyoshi ${ }^{1,3} \cdot$ Yasuhiro Miki $^{1} \cdot$ Kentaro Tamaki $^{1,2} \cdot$ Yoshihiko Kamada $^{2}$. \\ Kano Uehara ${ }^{2}$ - Seiko Tsuchiya ${ }^{2}$ - Shigeharu Terukina ${ }^{2}$. Erina Iwabuchi ${ }^{1}$ - Ayako Kanai ${ }^{1,4} \cdot$ Minoru Miyashita $^{4}$. \\ Takanori Ishida $^{4} \cdot$ Nobumitsu Tamaki $^{2} \cdot$ Hironobu Sasano ${ }^{1,3}$ (])
}

Published online: 4 October 2021

(c) Springer Science+Business Media, LLC, part of Springer Nature 2021

\section{Correction to: \\ Breast Cancer Research and Treatment (2021) 189:39-48 \\ https://doi.org/10.1007/s10549-021-06288-3}

In the original publication of the article, the antibody against GLP-1R which we used in this study was not mouse monoclonaly ab166987 as reported in the manuscript but rabbit polyclonal ab188602, both commercially obtained from Abcam.

Publisher's Note Springer Nature remains neutral with regard to jurisdictional claims in published maps and institutional affiliations.

The original article can be found online at https://doi.org/10.1007/ s10549-021-06288-3.

Hironobu Sasano

hsasano@patholo2.med.tohoku.ac.jp

1 Department of Pathology, Tohoku University Graduate

School of Medicine, 2-1 Seiryo-machi, Aoba-ku, Sendai,

Miyagi 980-8575, Japan

2 Department of Breast Surgical Oncology, Nahanishi Clinic,

Naha, Okinawa, Japan

3 Department of Pathology, Tohoku University Hospital,

Sendai, Miyagi, Japan

4 Department of Breast and Endocrine Surgical Oncology, Tohoku University Graduate School of Medicine, Sendai, Miyagi, Japan 